\title{
Immune Effects of Conventional Cancer Therapeutics
}

\author{
Sandra Demaria, Giulia Petroni, and Lorenzo Galluzzi
}

\section{KEY POINTS}

- Conventional chemotherapy, targeted anticancer agents, and radiotherapy have immunological effects that contribute to their activity in vivo.

- Immunological effects can be "on-target" (affecting cancer cells directly) or "off-target" (affecting immune cells).

- "On-target" immunostimulatory effects enhance immunogenicity of the cancer cells by improving antigenicity (increased expression of antigens or major histocompatibility complex [MHC] molecules) and/or adjuvanticity (generation of danger signals or damageassociated molecular patterns [DAMPs]).

- "Off-target" immunostimulatory effects are direct when the drug activates antitumor immune effectors or indirect when the drug eliminates or inhibits immunosuppressive cells.

- Cancer therapy can also mediate immunosuppressive effects as it promotes the accumulation or activation of regulatory and suppressor cells or factors.

- Treatments able to induce an immunogenic form of cancer cell death are often synergistic with immune checkpoint inhibitors.

- Rational combinations of conventional chemotherapy and/or radiotherapy with immunotherapy are undergoing clinical testing.

\section{INTRODUCTION}

Conventional cancer therapeutics, which include cytotoxic chemotherapy and radiotherapy, were developed with the goal of killing transformed cells while sparing normal cells as much as possible. An improved understanding of the molecular alterations that drive a specific cancer type has fostered the development of targeted therapeutics with increased selectivity and limited toxicity. ${ }^{1}$ Technological advances in imaging and radiation dose delivery have also improved tumor targeting by radiotherapy. ${ }^{2}$ At the same time, unequivocal demonstration that the malignant potential of transformed cells is regulated by the immune system fostered the development of anticancer therapeutics that target immune cells. ${ }^{3}$ The success of the latter approach urges revisiting long-held assumptions about the mechanisms through which conventional cancer therapeutics work and assessing their effects on cells of the immune system and on tumor-host interactions. ${ }^{4,5}$ This information becomes essential in the new era of immuno-oncology, in which several immune checkpoint inhibitors are approved for treatment of a growing list of cancer types and many more immunotherapies are likely to enter routine clinical care in the coming years. ${ }^{6}$ In this new landscape, the immunological effects of conventional and targeted therapeutics used to treat patients before, during, or after immunotherapy need to be clearly understood as they can profoundly influence disease outcome.

Conventional chemotherapy and radiation preferentially affect proliferating cells, a feature not only of cancer but also of cells of the adaptive immune system, and have been considered uniformly immunosuppressive. While this is true for some agents, especially when used

Authors' Disclosure: SD has received compensation for consultant/advisory services from Lytix Biopharma, Mersana Therapeutics, Genentech, and EMD Serono, and research support from Lytix Biopharma and Nanobiotix. GP has no relevant conflicts of interest to disclose. LG has received consulting fees from OmniSEQ, Astra Zeneca, Inzen, and the Luke Heller TECPR2 Foundation, and he is a member of the Scientific Advisory Committee of Boehringer Ingelheim, The Longevity Labs, Onxeo, and OmniSEQ. 
at high doses, experimental and clinical evidence accumulating over the past 15 years shows that many cytotoxic cancer treatments can stimulate antitumor immune responses and that the latter contribute significantly to their therapeutic activity. ${ }^{7-14}$ Immunostimulatory effects of chemotherapy can be categorized as "on-target" when they result directly from the interaction of the drug with cancer cells or "off-target" when they result from effects of the drug on immune cells (Figure 29.1). A similar definition applies to targeted therapeutics, at least in part to focal radiotherapy, since radiation affects all compartments of the tumor stroma including immune cells. For
A

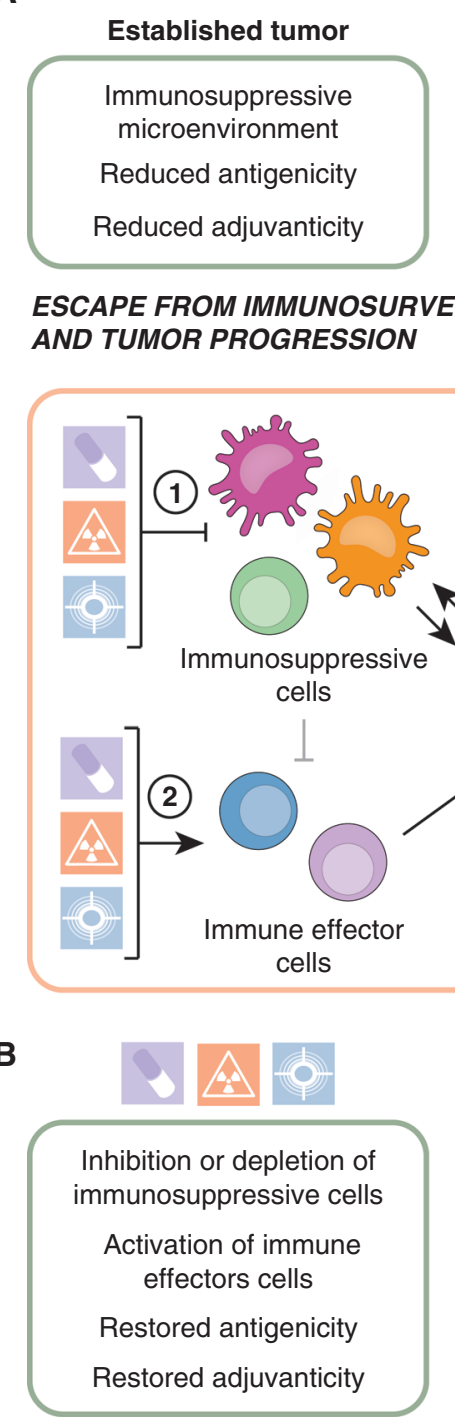

THERAPEUTICALLY RELEVANT IMMUNE RESPONSE

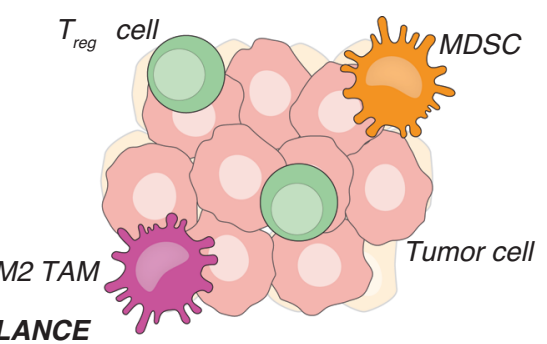

ILLANCE ons
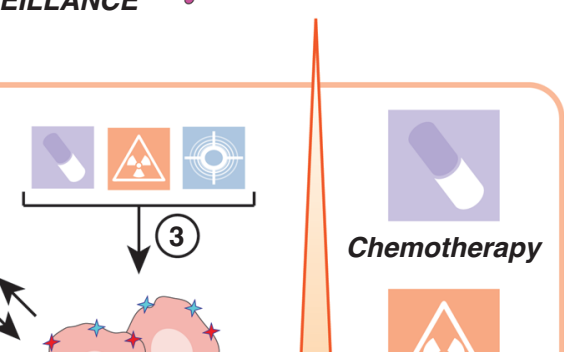
(3) (3)

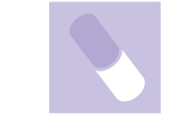

Chemotherapy

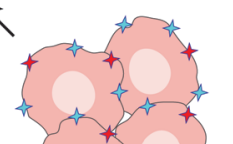

Tumor cells

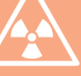

Radiation therapy

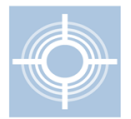

Targeted therapy
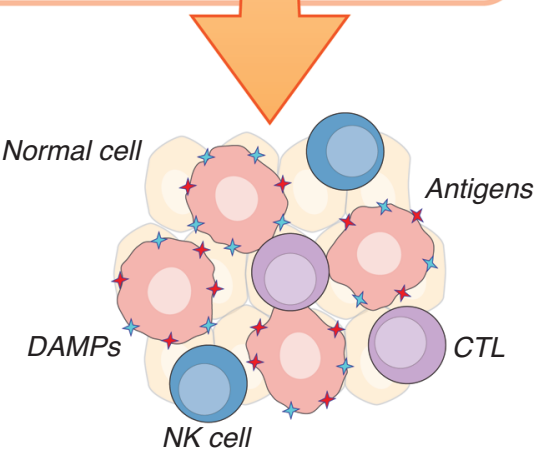

Figure 29.1 Immunostimulatory effects of conventional chemotherapeutics, targeted anticancer agents, and radiation therapy. According to current models, malignant precursors only form clinically manifest tumors as they escape immunosurveillance. Such an escape generally involves the selection of cancer cell variants with reduced immunogenicity (i.e., reduced antigenicity or reduced adjuvanticity) and/or the establishment of robust immunosuppressive mechanisms that operate locally (i.e., within the tumor microenvironment) and systemically (A). Multiple chemotherapeutics, targeted anticancer agents, and radiation therapy support the (re-)activation of tumor-targeting immune responses by (1) inhibiting or depleting immunosuppressive cell populations, (2) boosting the activity of immune effectors cells, and/or (3) restoring the antigenicity or adjuvanticity of neoplastic cells (B).

CTL, CD8+ cytotoxic lymphocyte; DAMPs, damage-associated molecular patterns; MDSC, myeloid-derived suppressor cell; NK, natural killer; TAM, tumor-associated macrophage; $\mathrm{T}_{\text {reg' }} \mathrm{CD}^{+} \mathrm{CD} 25^{+} \mathrm{Foxp}^{+}$regulatory $\mathrm{T}$. 
the purpose of discussion in this chapter we will categorize on-target and off-target effects as outlined next and refer the reader to Section I of this book for a more detailed description of the key components and mechanisms of antitumor immune responses.

\section{ON-TARGET IMIMUNE EFFECTS}

Tumors that become clinically evident have escaped immunological elimination by becoming less immunogenic or by suppressing antitumor immune effectors ${ }^{15,16}$ (Figure 29.1A). Some cytotoxic agents can trigger a number of molecular responses in cancer cells that restore (at least part of) their immunogenicity. The immunogenicity of cancer cells is determined by their antigenicity and adjuvanticity. "Antigenicity" involves the expression of proteins that are not covered by central or peripheral tolerance. ${ }^{16}$ Somatic mutations that accumulate in cancer cells are a major (but not the sole) source of the so-called tumor neoantigens. ${ }^{17,18}$ Alternative tumor-specific antigens can also originate from nonmutated proteins that are overexpressed and/or ectopically expressed, ${ }^{19}$ splice variants or lipids derived from noncanonical posttranscriptional or posttranslational events,$^{20}$ re-expression of latent proteins that are normally silenced by DNA methylation during development (e.g., the so-called "oncofetal antigens"), ${ }^{21}$ as well as proteins aberrantly expressed from normally noncoding genomic regions ${ }^{22}$ or endogenous retroelements. ${ }^{20}$ Such antigens must be presented to $\mathrm{CD} 8^{+}$ cytotoxic T lymphocytes (CTLs) in association with major histocompatibility complex class I (MHC-I) molecules. Thus, both the number of antigenic epitopes and MHC-I expression affect cancer cell antigenicity. "Adjuvanticity" refers to the expression or release of danger signals collectively known as "damage-associated molecular patterns" (DAMPs) ${ }^{23,24}$ For the purpose of the present discussion, DAMPs can be broadly subdivided into two (partially overlapping) groups: (a) cell death-associated DAMPs and (b) cell stress-associated DAMPs. ${ }^{24}$ A stressful cell death, as occurs when cells are infected or exposed to some cytotoxic agents that trigger the premortem activation of the DNA damage response (DDR), endoplasmic reticulum (ER) stress response, and autophagy, results in release of DAMPs that promote the recruitment and activation of dendritic cells (DCs) by binding to pattern recognition receptors (PRRs), and thus initiate the presentation of tumor-derived antigens to T cells. ${ }^{24,25}$ An instance of cell death associated with elevated antigenicity and considerable DAMP emission is therefore defined as immunogenic cell death (ICD). ${ }^{26}$ Molecules that are expressed by cells coping with (but surviving) therapy include ligands for natural killer (NK) cell-activating receptors like killer cell lectin-like receptor K1 (KLRK1, best known as NKG2D), ${ }^{27}$ as well as the death receptor FAS/CD95, and adhesion molecules that improve recognition and killing of the cancer cells by CTLs. ${ }^{28}$ Although these molecules do not interact with PRRs as bona fide DAMPs do, ${ }^{29}$ they contribute to the activation of the antitumor immune effectors and are therefore considered here as cell stressassociated DAMPs.

\section{OFF-TARGET IMMUNE EFFECTS}

Multiple immunosuppressive mechanisms operate locally (i.e., within the tumor microenvironment) and systemically to hinder tumor rejection by the immune system..$^{15}$ Inhibition of DCs, CTLs, and NK cells is mediated by suppressive cytokines, such as transforming growth factor beta 1 (TGF- $\beta 1$ ), and co-inhibitory receptors, like cytotoxic T lymphocyte associated protein 4 (CTLA-4), programmed cell death 1 (PD-1), ${ }^{3}$ and hepatitis A virus cellular receptor 2 (HAVCR2, best known as TIM-3), ${ }^{30}$ within a network of interactions with immune cells mediating tolerogenic and suppressive function such as regulatory $\mathrm{T}\left(\mathrm{T}_{\mathrm{reg}}\right)$ cells, myeloid-derived suppressor cells (MDSCs),

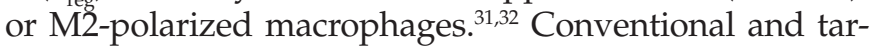
geted anticancer treatments have been shown to mediate immunostimulatory effects by direct interaction with immune effector cells or indirectly by eliminating or reprogramming immunosuppressive cells (Figure 29.1B).

\section{Conventional Chemotherapy}

\section{Increased Antigenicity}

Multiple chemotherapeutic agents currently employed in clinical practice have been found to boost the antigenicity of cancer cells by upregulating the levels of surface-expressed MHC-I molecules, an "on-target" immunostimulatory activity that likely contributes to the efficacy of these drugs. ${ }^{9}$ In combination with type I interferon (IFNI), 5-fluorouracil (an irreversible inhibitor of thymidylate synthase used for the treatment of several solid tumors) as well as doxorubicin (an anthracycline frequently employed in women with breast carcinoma) restored MHC-I expression on murine Panc02 pancreatic cancer cells, and this regimen had prominent therapeutic activity against Panc02 cells growing orthotopically in immunocompetent syngeneic mice. ${ }^{33,34}$ Gemcitabine (a nucleoside analogue mainly used for therapy of pancreatic carcinoma) had similar effects on human non-small cell lung carcinoma (NSCLC) A549 cells, human colorectal carcinoma HCT-116 cells, and human breast carcinoma MCF-7 cells. ${ }^{35}$ The upregulation of MHC-I molecules induced by gemcitabine was associated with (a) increased tumor-specific $T$ cell cytotoxicity in vitro, and this could be inhibited by an MHC-I-targeting monoclonal antibody ${ }^{35}$; and (b) with increased beta 2 microglobulin expression (which is required for MHC-I exposure), upregulation of immune-proteasomal subunits, and altered antigenic epitopes exposed by tumor cells. ${ }^{36}$ Along similar lines, 
cisplatin (a DNA-damaging agent platinum derivative approved for use in patients with a wide variety of solid tumors) employed at low doses efficiently upregulated surface-exposed MHC-I molecules in cultured mouse lung adenocarcinoma TC-1 cells and in human head and neck squamous cell carcinoma (HNSCC) UMSCC-46 and UMSCC-74A cell lines, ${ }^{37,38}$ as did oxaliplatin (another platinum-based DNA-damaging agent commonly used in combinatorial regimens for the treatment of colorectal cancer).$^{38}$ Moreover, MHC-I expression was upregulated by two topoisomerase inhibitors employed against different solid cancers, topotecan and irinotecan, in human breast carcinoma ZR-75-1 cells and murine mammary FM3A carcinoma cells, respectively, most likely as a consequence of autocrine/paracrine IFN-I signaling. ${ }^{39,40}$ At least theoretically, a wide panel of DNA-damaging agents, including radiation therapy (RT) employed at suboptimal doses (i.e., doses that are unable to promote cell death) ${ }^{41}$ could increase the antigenicity of cancer cells by favoring the generation of mutational neoantigens. ${ }^{17}$ These agents include cisplatin and other platinum derivatives (like carboplatin, which is frequently employed against ovarian carcinoma, and oxaliplatin, which is approved for the treatment of colorectal carcinoma) ${ }_{1}^{42}$ doxorubicin and other anthracyclines (such as idarubicin, which is also part of the chemotherapeutic options for acute myeloid leukemia [AML]), ${ }^{43}$ topotecan and other topoisomerase inhibitors (like etoposide, which is employed against multiple hematological and solid neoplasms), ${ }^{44}$ and alkylating agents (such as temozolomide, which is approved for use in glioblastoma and astrocytoma patients) ${ }^{45}$ However, a recent study of gliomas recurring after temozolomide-based chemotherapy demonstrates that hypermutated tumor cells do not necessarily become more sensitive to immunotherapy, ${ }^{46}$ lending further support to the emerging notion that neoantigen quality may be critical for immunogenicity. ${ }^{47-49}$

\section{Increased Adjuvanticity}

Multiple conventional chemotherapeutic agents mediate "on-target" immunostimulatory effects by increasing the capacity of malignant cells to emit danger signals as they respond to stress. ${ }^{9,50}$ Cell stress-associated DAMPs are emitted by malignant cells that successfully cope with the potentially lethal effects of chemotherapy, and hence survive treatment. ${ }^{51}$ Prototypic DAMPs of this class include various ligands for the activating NK-cell receptor NKG2D, including MHC-I polypeptiderelated sequence A (MICA), MHC-I polypeptide-related sequence $B(\mathrm{MICB})$, and retinoic acid early transcript 1E (RAET1E), which are upregulated on the membrane of cancer cells experiencing DNA damage. ${ }^{27}$ On the other hand, cell death-associated DAMPs are emitted by neoplastic cells that are unable to cope with the damaging effect of chemotherapy and eventually die as they attempt to do so (i.e., they activate adaptive stress responses that are insufficient for the recovery of homeostasis). ${ }^{23}$ DAMPs liberated following chemotherapy-induced cancer cell death trigger signaling cascades that ultimately result in the induction of adaptive immune responses. ${ }^{24,26,52}$ The optimal perception of ICD has been reported to involve the following cascade of events: (a) the exposure of calreticulin (CALR) and other ER chaperones on the cell surface, where they facilitate the phagocytosis of dead cells and corpses by antigen-presenting cells (APCs); ${ }^{53,54}$ (b) the secretion of ATP, which functions both as a chemotactic cue for myeloid cells and as a pro-inflammatory signal, ${ }^{55,56}$ (c) the release of annexin A1 (ANXA1), which guides the final approach of myeloid cells toward dying cells; ${ }^{57}(\mathrm{~d})$ the liberation of high-mobility group box 1 (HMGB1), a nuclear protein with multipronged immunostimulatory activity; ${ }^{58}(\mathrm{e})$ the activation of an autocrine/paracrine type I IFN signaling pathway, via cyclic GMP-AMP synthase (CGAS), culminating with the release of chemotactic cytokines, including $\mathrm{C}-\mathrm{X}-\mathrm{C}$ motif chemokine ligand 10 (CXCL10, a chemoattractant for T cells); ;9,60 and (f) passive release of nucleic acids which can engage Toll-like receptor 3 (TLR3), TLR7/8, and/or TLR9. ${ }^{60}$

Several chemotherapeutics have been shown to upregulate NKG2D ligands on the surface of cancer cells., ${ }^{9,24}$ 5-Fluorouracil as well as doxorubicin (both in combination with type I IFN) efficiently stimulated the exposure of RAET1E and UL16 binding protein 1 (ULBP1, which also activates NKG2D) on the surface of murine Panc02 cells maintained in vitro. ${ }^{33,34}$ Treatment with doxorubicin plus interleukin-12 (IL-12) also restored expression of the NKGD2 ligands MICA, MICB, RAET1E, and ULBP2 in vivo, in multiple murine tumors encompassing $4 \mathrm{~T} 1$ breast carcinomas, CT26 colorectal carcinomas, Lewis lung carcinomas (LLCs), and K7M3 osteosarcomas, as well as in a genetically engineered model of mammary carcinoma, which was coupled to CD8 ${ }^{+}$CTL-dependent tumor regression. ${ }^{61}$ Gemcitabine at doses not affecting cell proliferation upregulated surface-exposed MICA and MICB in two out of six human pancreatic cancer cell lines tested in vitro (i.e., PANC-1 and HPAF-II cells), but this failed to correlate with increased $\mathrm{T}$ cell cytotoxicity, possibly as a result of the concomitant release of soluble MICA and/or MICB. ${ }^{62}$ Gemcitabine also upregulated the expression of ULBP2, ULBP5, and ULBP6, as well as other NKG2D ligands, and of death receptor FAS/CD95 in human HCT-116 colorectal carcinoma cells and A549 NSCLC cells, and weakly increased the expression of FAS/CD95 on the surface of MCF7 breast carcinoma cells. ${ }^{63}$ Docetaxel (a taxane employed for the treatment of various carcinomas) promoted the exposure of MICA, MICB, ULBP1, and/or ULBP2 on cultured human breast carcinoma BT474 and MDAMB361 cells, 
as well as in MDAMB361 cells grown in SCID mice, which was accompanied by a $15 \%$ to $40 \%$ increase in NK cell-dependent antibody-mediated cellular cytotoxicity. ${ }^{64}$ Along similar lines, dacarbazine (a mainstay in the treatment of melanoma) robustly upregulated multiple NKG2D ligands including MICA, MICB, RAET1E, and/ or ULBP1 in cultured mouse melanoma B16F10 cells and human melanoma Mel-C cells (at both the mRNA and protein level), correlating with the ability of dacarbazine to improve the efficacy of checkpoint blockers in immunocompetent mice bearing B16F10 melanomas. ${ }^{65}$

In addition, multiple chemotherapeutics commonly employed in clinical practice have been shown to promote bona fide ICD, as monitored in gold-standard vaccination experiments involving immunocompetent mice and syngeneic tumors. ${ }^{66}$ These agents include (but may not be limited to) doxorubicin, idarubicin, and epirubicin (yet another anthracycline used for the treatment of breast carcinoma, most often in the context of combinatorial chemotherapeutic regimens) $)^{67-69}$; oxaliplatin ${ }^{70-74}$; cyclophosphamide (an alkylating agent approved for the therapy of hematological and solid neoplasms) ${ }^{75-77}$; bortezomib (a proteasome inhibitor used for the treatment of multiple myeloma) ${ }^{78}$; and pemetrexed (an antimetabolite approved for use against pleural mesothelioma and NSCLC). ${ }^{79}$ Recently, other chemotherapeutic agents that are under clinical development for cancer therapy, such as lurbinectedin (a synthetic alkaloid analogue) and PT-112 (a platinum-pyrophosphate conjugate), have been shown to induce several markers of ICD in different tumor models. ${ }^{80,81}$ Of note, ICD is accompanied by such an adjuvanticity that chemotherapeutic ICD inducers appear to synergize with checkpoint blockers to induce potent therapeutic responses even in tumors that are normally insensitive to checkpoint blockade. ${ }^{73,79-85}$ A considerable number of clinical trials is currently focusing on the safety and efficacy of ICD-inducing chemotherapeutics plus immune checkpoint blockers (ICBs) in patients affected by a variety of solid and hematological tumors. ${ }^{86,87}$

\section{Direct Immunostimulation}

Although it may seem counterintuitive, some conventional chemotherapeutics mediate "off-target" immunostimulatory effects by delivering activating signals to the myeloid cell populations that initiate anticancer immune responses and/or to lymphoid immune effectors. ${ }^{9}$ Thus, while dose-intensive cyclophosphamide has been employed for decades as part of myelo- and lymphoablative preconditioning (to prepare patients for hematopoietic stem cell transplantation) ${ }^{88}$ metronomic cyclophosphamide has multipronged immunostimulatory effects. Low-dose cyclophosphamide has been shown to support therapeutically relevant Th1 and Th17 responses in immunocompetent mice developing $\mathrm{KRAS}^{\mathrm{G} 12 \mathrm{D}}$-driven lung cancer in the absence of functional p53 through a gut microbiota-dependent mechanism. ${ }^{89}$ This regimen promoted CTL activity and the expansion of circulating NK cells in C57BL/6 mice implanted with syngeneic GL261 gliomas, an effect that contributed to the efficacy of treatment. ${ }^{76}$ Along similar lines, metronomic cyclophosphamide synergized with a tumor lysate-based vaccine in the eradication of aggressive mouse AgN2a neuroblastomas growing in syngeneic hosts, an activity that also depended on $\mathrm{CD} 8^{+} \mathrm{CTL}$ expansion. ${ }^{90}$ In combination with radiotherapy, 5-fluorouracil promoted tumor infiltration by CD8 $8^{+}$CTLs in a cohort of 52 locally advanced rectal cancer patients, ${ }^{91}$ and an increased number of $\mathrm{CD}^{+}$tumor-infiltrating lymphocytes (TILs) expression have also been found in a cohort of 69 patients with esophageal squamous cell carcinoma treated with 5-fluorouracil and cisplatin. This effect has been linked to compensatory upregulation of the immunosuppressive ligand CD274 (best known as PD-L1). ${ }^{92}$ Of note, other chemotherapeutic agents can upregulate the expression of PD-L1 on cancer cells or tumor-infiltrating myeloid cells in preclinical ${ }^{85}$ and clinical settings, ${ }^{93-96}$ most likely reflecting increased tumor infiltration by $\mathrm{CD}^{+}$CTLs. Moreover, low-dose 5-fluorouracil enabled a peptide-based anticancer vaccine to mediate therapeutic effects in immunocompetent mice bearing syngeneic E.G7 lymphoma cells, an effect that was linked to enhanced CD8 ${ }^{+}$CTL activity. ${ }^{97}$

In a cohort of 28 pancreatic cancer patients, gemcitabine administration was associated with considerably increased numbers of circulating CD11 $\mathrm{c}^{+} \mathrm{DCs}$ and CD14 ${ }^{+}$ monocytes (both of which are known to contribute to natural and therapy-elicited anticancer immunosurveillance). ${ }^{98,99}$ Similarly, gemcitabine restored defective cross-presentation by tumor-infiltrating DCs in a model of mouse AB1 mesothelioma engineered to express an exogenous immunoreactive antigen, ${ }^{100,101}$ as did paclitaxel (a taxane used for the treatment of multiple solid tumors) in mice bearing transgenic breast carcinomas. ${ }^{102}$ Oxaliplatin potently boosted the anticancer activity of neutrophils and macrophages in immunocompetent mice bearing syngeneic EL4 lymphomas via a microbiota-dependent circuitry, thus resembling metronomic cyclophosphamide. ${ }^{103}$ Moreover, oxaliplatin also favored the activation of DCs by increasing IL-12 production and concomitantly decreasing PD-L1 expression by DCs themselves. ${ }^{104}$ Neoadjuvant paclitaxel favored the accumulation of TILs in a cohort of 25 breast carcinoma patients, correlating with clinical responses to treatment. ${ }^{105}$ Finally, pemetrexed stimulated IFN- $\gamma$ production by circulating NK cells from pancreatic cancer patients, an immunostimulatory effect that was abrogated by concurrent gemcitabine treatment and associated with the depletion of $\mathrm{CD} 5 \mathrm{RO}^{+}$memory $\mathrm{T}$ cells. ${ }^{106}$ Pemetrexed also increased $\mathrm{T}$ cell activation in vitro and in preclinical 
models of colorectal carcinoma by simultaneously inducing ICD and mediating direct "on-target" effects on T cells. ${ }^{79}$ Taken together, these observations suggest that the direct immunostimulatory effects of specific chemotherapeutics may be sensitive to contextual variables including the presence of additional drugs.

\section{Indirect Immunostimulation}

Arguably the most common mechanism through which conventional chemotherapeutics employed at metronomic doses promote therapeutically relevant immunostimulation is by inhibiting or depleting immunosuppressive cell populations. ${ }^{9}$ Thus, it appears possible to find doses and/or schedules of chemotherapeutic agents that preferentially deplete immunosuppressive cells over immune effector cells in vivo. Molecular mechanisms are understood in only a subset of cases. Human $\mathrm{CD}^{+} \mathrm{CD} 25^{+} \mathrm{Foxp}^{+} \mathrm{T}_{\text {reg }}$ cells have been shown to be particularly sensitive to cyclophosphamide-driven cell death as they do not express ATP-binding cassette, subfamily $\mathrm{B}$ (MDR/TAP), member 1 (ABCB1), which normally extrudes the active metabolite of the drug. ${ }^{107}$ On the contrary, why other immunosuppressive cells succumb so easily in response to a wide panel of chemotherapeutic agents employed at low doses remains unknown. ${ }^{9}$

Metronomic cyclophosphamide has been reported to preferentially deplete $\mathrm{CD} 4^{+} \mathrm{CD} 25^{+} \mathrm{Foxp} 3^{+} \mathrm{T}_{\text {reg }}$ cells in nine end-stage cancer patients, correlating with the restoration of NK-cell and CD8 ${ }^{+}$CTL activity. ${ }^{108} \mathrm{~A}$ similar effect has been seen in C57BL/6 mice bearing syngeneic GL261 gliomas ${ }^{76}$ and in rats bearing syngeneic colorectal carcinomas. ${ }^{109}$ Conversely, in mice bearing syngeneic 4T1 breast carcinoma cells and A20 B-cell lymphoma cells, low-dose metronomic cyclophosphamide slightly increased circulating $\mathrm{T}_{\mathrm{reg}}$ cells as it decreased circulating B and effector T cells. ${ }^{110}$ Despite these latter observations, cyclophosphamide and an anti-PD-L1 antibody synergized in the control of 4T1 and A20 tumors. ${ }^{110}$ A $\mathrm{T}_{\text {reg }}$ cell-depleting activity has also been ascribed to docetaxel, in a cohort of 40 NSCLC patients; ${ }^{111}$ gemcitabine, in 53 subjects with pancreatic cancer ${ }^{112}$ and in 40 NSCLC patients (receiving gemcitabine plus cisplatin), ${ }^{113}$ and vinorelbine (a vinca alkaloid employed for the treatment of solid tumors), in 14 NSCLC patients treated with this agent in combination with cisplatin. ${ }^{114}$ 5-Fluorouracil was reported to deplete circulating MDSCs from immunocompetent mice bearing carcinogen-driven colorectal cancer ${ }^{115}$ or syngeneic EL4 lymphomas, ${ }^{116}$ as well as from 23 patients with stage IV colorectal carcinoma, ${ }^{116}$ an indirect immunostimulatory effect that was abolished by the concomitant administration of irinotecan but not oxaliplatin. ${ }^{115}$ In mice bearing FM3A breast carcinomas, irinotecan depleted $\mathrm{T}_{\mathrm{reg}}$ cells when used as monotherapy, but an increase in $\mathrm{CD} 8^{+} \mathrm{T}$ cells in tumors or lymph nodes was only observed when used in combination with an antiPD-L1 antibody. ${ }^{40}$ Finally, doxorubicin could increase the percentage of CTLs secreting IFN- $\gamma$ through reducing the accumulation of $\mathrm{T}_{\text {reg }}$ cells in LLC and 4T1 breast carcinomas only when coadministered with IL-12, while the administration of doxorubicin alone resulted in $\mathrm{T}_{\text {reg }}$ cell accumulation in the same models. ${ }^{117}$

Gemcitabine caused a decrease in the percentage of circulating MDSCs in a variety of syngeneic tumor models, including CT26 colorectal carcinomas, ${ }^{118}$ A20 lymphomas, ${ }^{119}$ WEHI-164 fibrosarcomas, ${ }^{120}$ AB12 mesotheliomas, ${ }^{121}$ L1C2 bronchoalveolar carcinomas, ${ }^{121}$ and K7M2 osteosarcomas ${ }^{120}$ established in BALB/c mice, as well as LLC or TC-1 lung adenocarcinomas, ${ }^{121,122}$ L1C2 bronchoalveolar carcinomas, ${ }^{121}$ AE-17 mesothelioma, ${ }^{121}$ and EL4 lymphoma ${ }^{116}$ established in C57BL/6 mice. Along similar lines, paclitaxel was capable of depleting circulating MDSCs from mice bearing transgene-driven melanoma, accounting for most of the therapeutic efficacy of the drug in this setting. ${ }^{123}$ Moreover, low levels of MDSCs have been found in the peripheral blood of patients with gastric cancer treated with paclitaxel. ${ }^{124}$

Finally, the experimental platinum-pyrophosphate conjugate PT-112 has been linked to a reduction in immunosuppressive cells (i.e., MDSCs, tumor-associated macrophages, and $\mathrm{T}_{\text {reg }}$ cells) and a concomitant increase in CD8 ${ }^{+}$CTLs in the tumor microenvironment of CT26 colon carcinomas. ${ }^{81} \mathrm{~A}$ similar indirect immunostimulatory effect has been ascribed to oxaliplatin, which reduced peritoneal metastasis by decreasing the number of tumor-associated macrophages and MDCSs in the spleen of BALB/c mice bearing syngeneic CT26 colon carcinomas, ${ }^{125}$ and to a nanoparticle formulation of doxorubicin that causes the repolarization of murine M2-polarized immunosuppressive tumor-associated macrophages into their M1-polarized tumoricidal counterparts in 4T1 mammary carcinoma established in BALB/c mice. ${ }^{126}$ Interestingly, gemcitabine and paclitaxel have also been shown to cause the M2-to-M1 repolarization of human and murine macrophages in vitro and in tumor samples from ovarian cancer patients. ${ }^{127,128}$ However, it remains unclear to which extent such a conversion occurs in pancreatic cancer patients treated with gemcitabine-based chemotherapy in vivo, and whether such indirect immunostimulatory effects are therapeutically relevant in the clinic. Irrespectively, these observations corroborate the notion that a wide panel of chemotherapeutics currently employed in clinical practice can inhibit immunosuppressive cell populations, especially when employed according to metronomic regimens.

Overall, these data highlight the multiple effects of conventional chemotherapeutics on the antitumor host response (Table 29.1). While it remains to be established to which degree the therapeutic response to chemotherapy in patients is the result of direct tumor cytotoxicity 
Table 29.1 Immunological Effects of Conventional Chemotherapy (Examples)

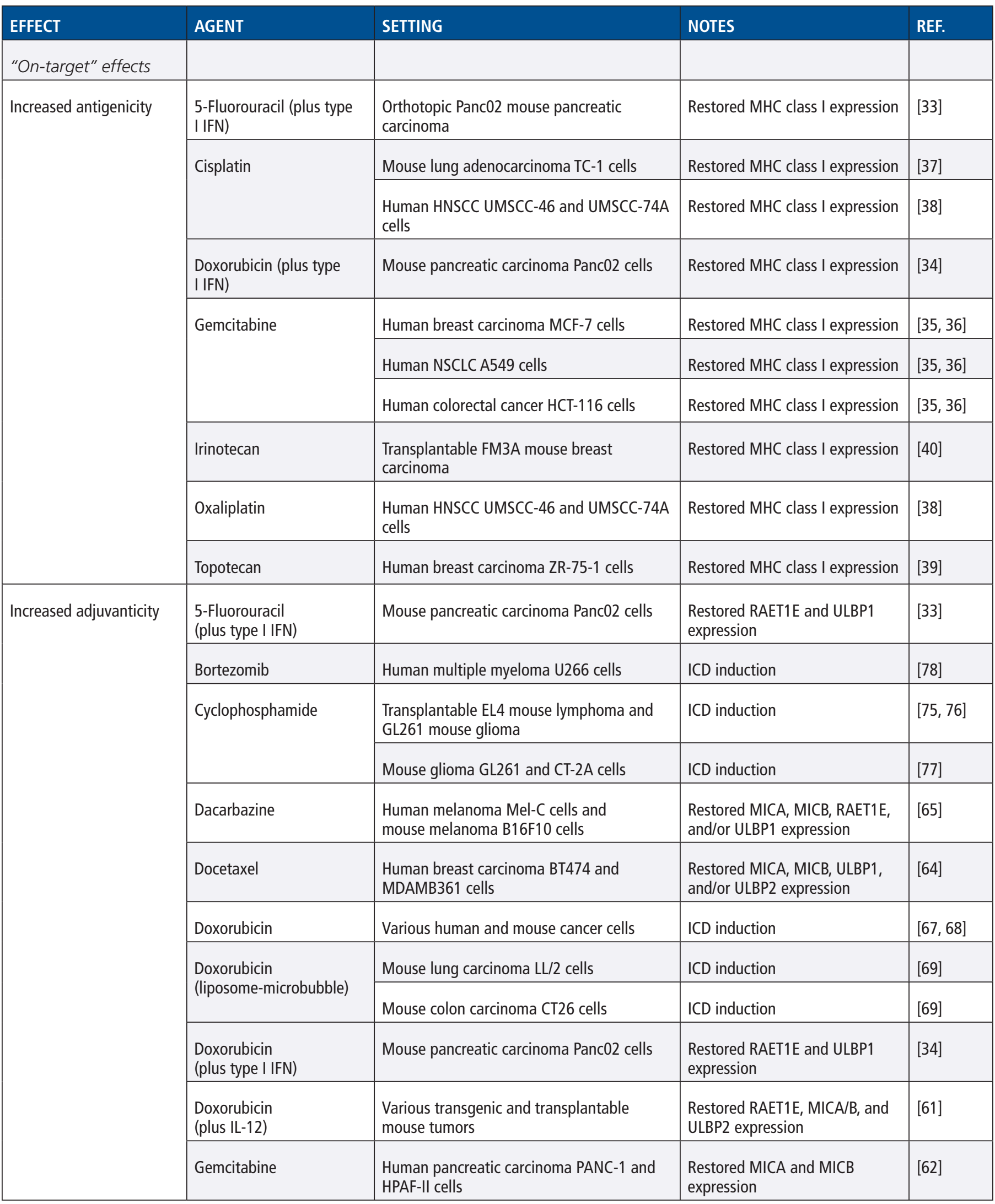


Table 29.1 Immunological Effects of Conventional Chemotherapy (Examples) (continued)

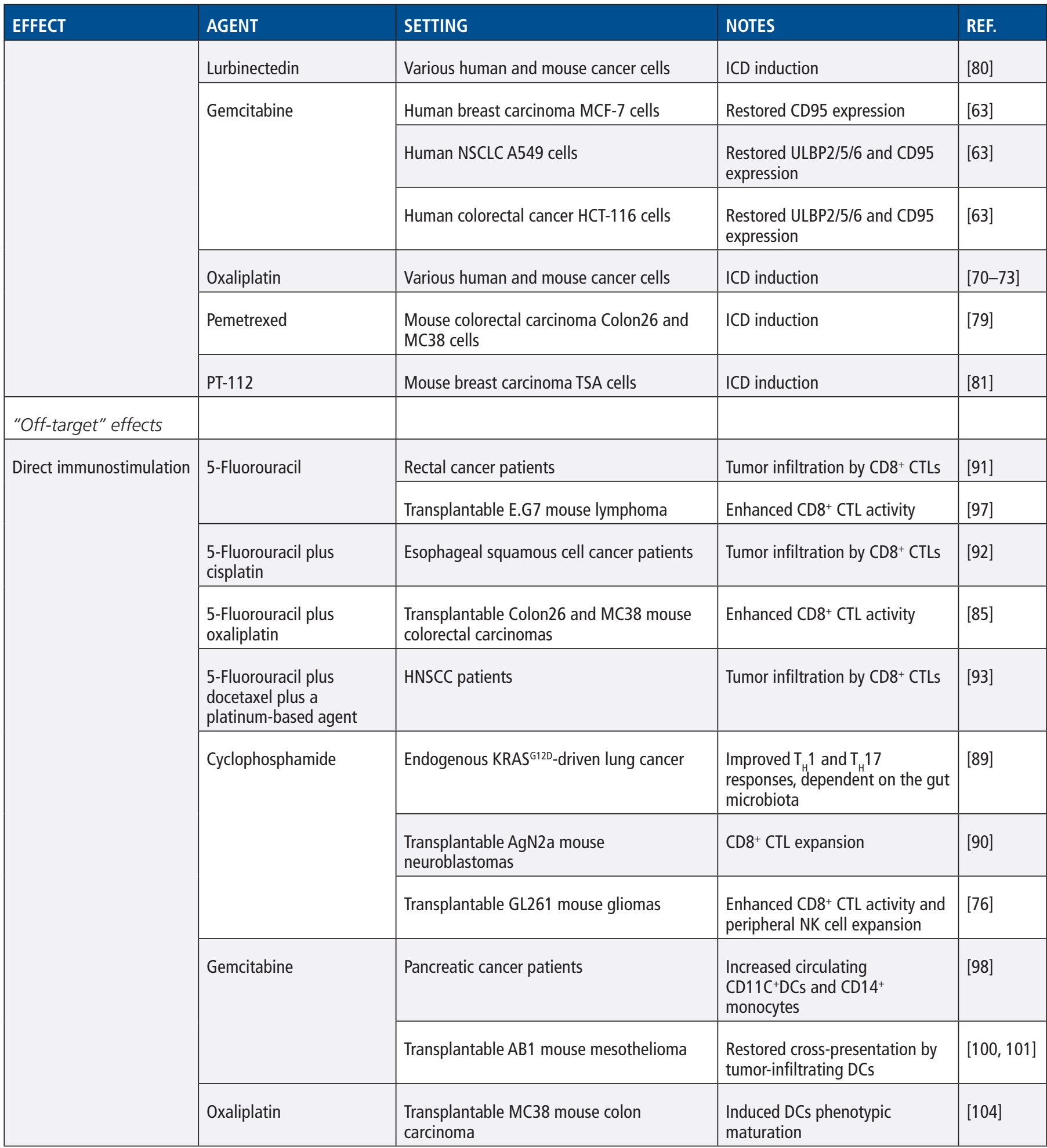


Table 29.1 Immunological Effects of Conventional Chemotherapy (Examples) (continued)

\begin{tabular}{|c|c|c|c|c|}
\hline EFFECT & AGENT & SETTING & NOTES & REF. \\
\hline & \multirow[t]{2}{*}{ Paclitaxel } & Breast carcinoma patients & $\begin{array}{l}\text { Increased levels of TILs, } \\
\text { correlating with clinical } \\
\text { responses }\end{array}$ & [105] \\
\hline & & Transgenic mouse breast carcinoma & $\begin{array}{l}\text { Restored cross-presentation by } \\
\text { tumor-infiltrating DCs }\end{array}$ & [102] \\
\hline & \multirow[t]{2}{*}{ Pemetrexed } & Pancreatic cancer patients & $\begin{array}{l}\text { Enhanced IFN- } \gamma \text {-secretory } \\
\text { activity in circulating NK cells }\end{array}$ & [106] \\
\hline & & $\begin{array}{l}\text { Primary T cells and transplantable Colon26 } \\
\text { and MC } 38 \text { mouse colon carcinomas }\end{array}$ & $\begin{array}{l}\text { Activated T cells and enhanced } \\
\text { CD8 } 8^{+} \text {CTL activity }\end{array}$ & [79] \\
\hline \multirow{16}{*}{$\begin{array}{l}\text { Indirect } \\
\text { immunostimulation }\end{array}$} & \multirow[t]{3}{*}{ 5-Fluorouracil } & Colorectal carcinoma patients & Depletion of circulating MDSCs & [115] \\
\hline & & Mouse carcinogen-driven colorectal cancer & $\begin{array}{l}\text { Depletion of MDSCS, abolished } \\
\text { by irinotecan coadministration }\end{array}$ & [115] \\
\hline & & Transplantable EL4 mouse lymphoma & Depletion of circulating MDSCs & [116] \\
\hline & \multirow[t]{3}{*}{ Cyclophosphamide } & Advanced cancer patients & $\begin{array}{l}\text { Depletion of } \mathrm{T}_{\text {reg }} \text { cells, linked to } \\
\text { NK cell and CD8 } 8^{+} \text {CTL activity }\end{array}$ & [108] \\
\hline & & Transgenic rat colorectal carcinoma & Depletion of $\mathrm{T}_{\text {reg }}$ cells & [109] \\
\hline & & Transplantable GL261 mouse glioma & Depletion of $\mathrm{T}_{\text {req }}$ cells & [76] \\
\hline & Docetaxel & NSCLC patients & Depletion of $\mathrm{T}_{\text {req }}$ cells & [111] \\
\hline & $\begin{array}{l}\text { Doxorubicin } \\
\text { (plus IL-12) }\end{array}$ & $\begin{array}{l}\text { Transplantable LLC lung and } 4 \mathrm{~T} 1 \text { breast } \\
\text { carcinomas }\end{array}$ & $\begin{array}{l}\text { Depletion of } \mathrm{T}_{\text {reg }} \text { cells linked to } \\
\text { CD8+ }{ }^{+} \text {CTL activity }\end{array}$ & [117] \\
\hline & $\begin{array}{l}\text { Doxorubicin } \\
\text { (nanoparticles) }\end{array}$ & Transplantable $4 \mathrm{~T} 1$ breast carcinoma & $\begin{array}{l}\text { M2-to-M1 repolarization linked } \\
\text { to CD8 } 8^{+} \text {CTL activity }\end{array}$ & [126] \\
\hline & \multirow[t]{4}{*}{ Gemcitabine } & Pancreatic cancer patients & Depletion of $\mathrm{T}_{\text {reg }}$ cells & [112] \\
\hline & & Human M2-polarized macrophages & M2-to-M1 repolarization & [127] \\
\hline & & $\begin{array}{l}\text { Transplantable K7M2 mouse osteosarcomas } \\
\text { and WEHI-164 mouse fibrosarcomas }\end{array}$ & Depletion of $\mathrm{T}_{\mathrm{reg}}$ cells & [120] \\
\hline & & Various transplantable mouse tumors & Depletion of circulating MDSCs & $\begin{array}{l}{[116,} \\
118-122]\end{array}$ \\
\hline & $\begin{array}{l}\text { Gemcitabine (plus } \\
\text { cisplatin) }\end{array}$ & NSCLC patients & Depletion of $\mathrm{T}_{\mathrm{reg}}$ cells & [113] \\
\hline & Irinotecan & $\begin{array}{l}\text { Transplantable FM3A mouse breast } \\
\text { carcinoma }\end{array}$ & Depletion of $\mathrm{T}_{\text {reg }}$ cells & [40] \\
\hline & Oxaliplatin & Transplantable CT26 mouse colon carcinoma & $\begin{array}{l}\text { Depletion of TAMs and of } \\
\text { MDCSs in the spleen, linked to } \\
\text { CD8 } 8^{+} \text {CTL activity }\end{array}$ & [125] \\
\hline
\end{tabular}


Table 29.1 Immunological Effects of Conventional Chemotherapy (Examples) (continued)

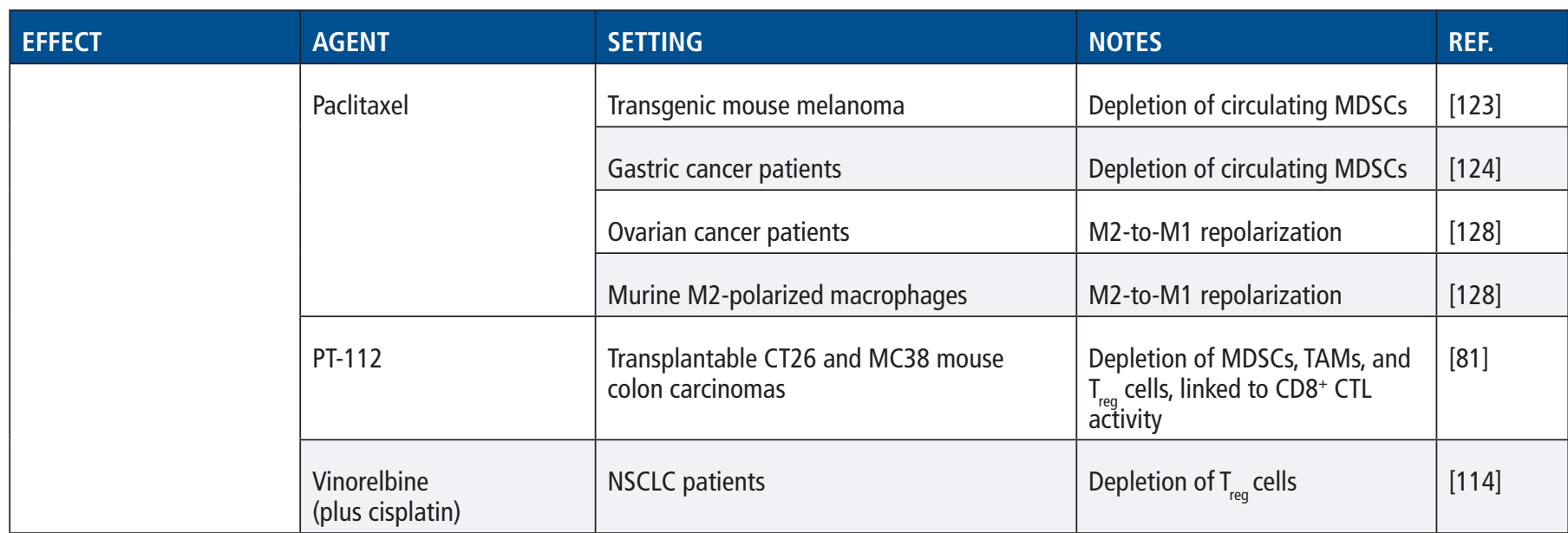

CTL, cytotoxic T lymphocyte; DC, dendritic cell; HNSCC, head and neck squamous cell carcinoma; ICD, immunogenic cell death; IFN, interferon; LCC, Lewis lung carcinoma; MDSC, myeloid-derived suppressor cell; MHC, major histocompatibility complex; MICA, MHC class I polypeptide-related sequence A; MICB, MHC class I polypeptide-related sequence B; NK, natural killer; NSCLC,

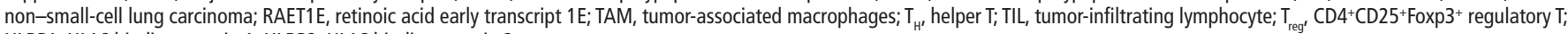
ULBP1, UL16 binding protein 1; ULBP2, UL16 binding protein 2.

Source: Modified with permission from Galluzzi L, Buqué A, Kepp 0, et al. Immunological effects of conventional chemotherapy and targeted anticancer agents. Cancer Cell. 2015;28(6):690-714. doi:10.1016/j.ccell.2015.10.012

versus the induction of antitumor immunity, it is clear that the immunomodulatory effects of a given chemotherapy can influence its interaction with immunotherapeutic agents, either positively or negatively.

\section{Targeted Therapy}

Similar to conventional chemotherapy, targeted anticancer agents can have unintended immunomodulatory effects that are the result of their on-target action (inhibition of a mutated or overexpressed oncogene product) or may result from the interaction of the drug with other targets expressed by immune cells. These effects can have positive or negative influences on antitumor immune responses and are important yet incompletely understood determinants of clinical efficacy. In support of this notion, in a mouse model of oncogene addiction, durable responses to specific targeting of an oncogene were shown to require the contribution of T cells. ${ }^{129}$

The tyrosine kinase inhibitor (TKI) imatinib mesylate developed against the oncogenic fusion protein BCR$\mathrm{ABL}$ is the prototype-targeted anticancer therapeutic and one of the most successful clinically. Initially developed for $\mathrm{BCR}-\mathrm{ABL}^{+}$chronic myelogenous leukemia (CML), ${ }^{130}$ imatinib was later shown to also have activity against the oncogenic kinase KIT, which is expressed by gastrointestinal stromal tumors (GISTs), ${ }^{131}$ and platelet-growth factor receptor beta (PDGFRB), which is expressed by several solid tumors. ${ }^{132}$ Induction of antileukemia T cell responses was reported in nine of $14 \mathrm{CML}$ patients in remission after imatinib treatment. ${ }^{133}$ In another study, BCR-ABL-specific $\mathrm{T}$ cells were detected in 10 of $10 \mathrm{CML}$ patients in maintenance with imatinib, more frequently in the bone marrow than in the peripheral blood. There was a correlation between BCR-ABL-specific T cells and lower minimal residual disease, and absence at leukemia relapse. ${ }^{134}$ Moreover, ibrutinib, which targets Bruton tyrosine kinase, has been reported to increase diversification of the T cell compartment by increasing the TCR repertoire diversity in patients with chronic lymphocytic leukemia. ${ }^{135}$

An impact of kinase targeting on host immune responses has also been observed in the context of GIST. In a mouse model of GIST driven by an activated KIT mutation, the therapeutic activity of imatinib was shown to be partially dependent on antitumor $\mathrm{CD} 8^{+} \mathrm{T}$ cells. The activation of antitumor immunity was due to downregulation of the immunosuppressive enzyme indoleamine 2,3-dioxygenase 1 (IDO1) in cancer cells, resulting in reduced $\mathrm{T}_{\mathrm{reg}}$ cells and increased effector CTL infiltration into the tumor, a change that correlated with imatinib sensitivity in human GISTs. ${ }^{136}$ Thus, activation of antitumor immunity is an on-target effect of imatinib in GISTs, which can be categorized as enhancing their adjuvantic$i t y$. In contrast, an off-target inhibitory activity of this drug against colony-stimulating factor 1 receptor (CSF1R) was likely responsible for decreased tumor-associated macrophage (TAM) recruitment to imatinib-treated GISTs. Despite such reduction, TAM interaction with apoptotic cancer cells in treated tumors was shown to promote M2 polarization, resulting in increased immunosuppression. ${ }^{137}$ Consistent with this data, coadministration of imatinib and an anti-CD40 antibody activated TAMs and resulted in superior antitumor activity. ${ }^{138}$ In other 
studies, the ability of imatinib to inhibit KIT on DCs was shown to promote DC-mediated NK-cell activation, culminating in antitumor effects against imatinibresistant GISTs. ${ }^{139,140}$ Imatinib also induced the M2-to-M1 repolarization of macrophages by TLR engagement and the M1-mediated NK-cell activation in neuroblastoma and CML samples, as did the imatinib-like drug nilotinib. ${ }^{141}$ Recent findings also demonstrate that imatinib can augment antitumor immunity by inducing selective depletion of $\mathrm{T}_{\text {reg }}$ cells and increasing the number of effector $\mathrm{CD}^{+} \mathrm{T}$ cells in patients with CML or melanoma. Mechanistically, imatinib appears to inhibit the lymphocyte-specific protein tyrosine kinase LCK protooncogene, Src family tyrosine kinase (LCK), which is highly expressed by $\mathrm{T}$ cells other than $\mathrm{T}_{\text {reg }}$ cells, rendering the latter more sensitive to apoptosis upon TCR inhibition in the presence of imatinib. ${ }^{142}$ The analysis of samples from dermatofibrosarcoma patients after treatment with imatinib also demonstrated that PDGFRB inhibition favors antigen presentation, linked to enhanced infiltration of the tumor by $\mathrm{T}$ cells and strong PD-L1 expression on the malignant cells themselves. ${ }^{143}$ Consistent with this, coadministration of PD-1/PD-L1 blockers correlated with superior therapeutic activity in a model of GISTs. ${ }^{144}$ ICBs also synergized with crizotinib, a TKI used to treat NSCLC, which acted as a potent ICD stimulator when combined with non-ICD-inducing chemotherapeutics like cisplatin, and induced offtarget effects by promoting immune infiltration in NSCLC tumors established in immunocompetent mice. ${ }^{145}$ These data highlight the complex and unsuspected effects of a molecularly targeted drug on the intended and unintended targets, which contribute to, and in some circumstances could hinder, the therapeutic response via immunological mechanisms. ${ }^{132}$

Many other TKIs used in the clinical practice for the treatment of different malignancies have been found to mediate direct and indirect effects on the immune system. ${ }^{9}$ For example, dasatinib, a second-generation TKI active against BCR-ABL, SRC, KIT, PDGFR, and ephrin tyrosine kinases, reduced the levels of MDSCs and $\mathrm{T}_{\text {reg }}$ cells in a mouse melanoma model, leading to improved $\mathrm{T}$ cell responses to a tumor vaccine, an offtarget indirect immunostimulatory effect. ${ }^{146} \mathrm{~A}$ similar reduction of MDSCs and $\mathrm{T}_{\text {reg }}$ cells was reported for sorafenib, another broad-spectrum TKI, in mice and patients with renal cell carcinoma, ${ }^{147,148}$ and sunitinib, which also upregulated CXCL10 and CXCL11 on the tumor endothelium, improving the recruitment of $\mathrm{T}$ cells in mouse melanoma and hepatocellular models, effects that are at least in part the consequence of the antiangiogenic activity of these drugs. ${ }^{149,150}$ Erlotinib and gefitinib, which target epidermal growth factor receptor (EGFR), were shown to increase the expression of NKG2D ligands on lung cancer cells, increasing their susceptibility to NK cell-mediated lysis, an example of an on-target increase in adjuvanticity. ${ }^{151}$ Off-target immunostimulatory effects have also been reported for lenvatinib, which showed potent antitumor activity in the tumor microenvironment of murine colorectal and hepatocellular tumors, characterized by reduced TAMs and increased percentage of CTLs secreting IFN- $\gamma$ and granzyme B. Moreover, combination treatment of lenvatinib plus anti-PD-1 further increased these effects resulting in greater antitumor activity. ${ }^{152,153}$

Activating mutations in B-Raf proto-oncogene (BRAF), encoding a serine/threonine kinase of the mitogen-activated protein kinase (MAPK) pathway, are frequent in melanoma and can be successfully targeted by specific BRAF inhibitors vemurafenib, encorafenib, and dabrafenib, alone or in combination with inhibitors of downstream mitogen-activated protein kinase kinase 7 (MAP2K7, best known as MEK, such as trametinib, cobimetinib, and binimetinib), but responses are generally short-lived. ${ }^{154,155}$ Treatment of melanoma cells with BRAF and MEK inhibitors was shown to increase expression of melanocyte differentiation antigens, resulting in improved recognition by $\mathrm{T}$ cells, an on-target increase in cancer cell immunogenicity. In a recent study, combined inhibition of BRAF and MEK also led to DC activation following the release of HMGB1 by melanoma cells. ${ }^{156}$ Although a previous study demonstrated that MEK inhibitor can impair $\mathrm{T}$ cell function, ${ }^{157}$ MEK inhibition has recently been linked to a blockage of naïve $\mathrm{CD} 8^{+} \mathrm{T}$ cell expansion and priming but an increased number of antigen-specific effector CD8 ${ }^{+}$ $\mathrm{T}$ cells in the tumor microenvironment (TME), and limited $\mathrm{T}$ cell exhaustion in vitro upon TCR stimulation. ${ }^{158}$ These findings have been confirmed in mouse models of AML as well as in primary samples of AML patients sensitive to trametinib. ${ }^{159}$ Moreover, the MEK inhibitor selumetinib combined with the PD-L1 blocker atezolizumab augmented antitumor immunity by upregulating MHC-I expression, boosting the production of several cytokines (i.e., IFN- $\gamma$, IL-6, IL-1 $\beta$, and TNF), and reducing PD-L1 expression in a panel of NSCLC cell lines. ${ }^{160}$ Other studies also suggest that $\mathrm{T}$ cell impairment may not be significant in vivo since patients co-treated with BRAF and MEK inhibitors showed increased infiltration of the tumor by $\mathrm{T}$ cells, which was associated with increased expression of PD-L1 (which is reflective of local IFN- $\gamma$ secretion). ${ }^{161,162}$ Importantly, responses to BRAF inhibitors correlated with the presence of an oligoclonal CD8 ${ }^{+}$ $\mathrm{T}$ cell infiltrate in pretreatment biopsies, suggesting a contribution of the immune system to clinical responses, while reduced levels of intratumoral $\mathrm{CD}^{+} \mathrm{T}$ cells were seen at progression. ${ }^{163,164}$ Interestingly, BRAF inhibition has also been shown to have off-target direct immunostimulatory effects on mouse and human NK cells, leading to increased phosphorylation of ERK1/2, CD69 upregulation, and improved effector functions. In a mouse model of $\mathrm{BRAF}^{\mathrm{V} 600 \mathrm{E}}$-driven melanoma, $\mathrm{NK}$ cells and perforin 
1 (PRF1) were required for therapeutic effects of BRAF inhibitors. ${ }^{165}$ Moreover, BRAF and MEK inhibitors combined with a PD-1 blocker decreased TAM and $\mathrm{T}_{\text {reg }}$ cell accumulation, improved IFN- $\gamma$ release, and enhanced antigen presentation in a preclinical melanoma model, and increased the frequency of long-lasting antitumor responses in patients with BRAF-mutated melanoma. ${ }^{166}$

Improved antitumor immunity and synergy with ICBs has been described for MEK inhibitors in different preclinical models of tumors driven by mutant KRAS proto-oncogene, GTPase (KRAS). ${ }^{167,168}$ Activating mutations of KRAS, which impact several signaling pathways including BRAF-MEK signaling and sustain carcinogenesis as well as treatment resistance in multiple oncological settings, ${ }^{169}$ have recently been considered as novel targets to impair tumor growth and enhance antitumor immunity. Interestingly, pharmacological inhibition of KRASG12C with AMG510 resulted in tumor regression linked to the activation of ICD in several KRAS ${ }^{\mathrm{G} 12 \mathrm{C}}$-driven tumor models. In addition, AMG510 improved the therapeutic efficacy of MEK inhibitors and a PD-1 blocker in KRAS ${ }^{\mathrm{G} 12 \mathrm{C}}$-expressing mouse colorectal tumors growing in immunocompetent syngeneic mice. ${ }^{170,171}$

Some of the therapeutics targeting growth factor receptors that are overexpressed by cancer cells, including HER2/ERBB2 and EGFR, are monoclonal antibodies (e.g., trastuzumab, cetuximab). Not surprisingly, these agents not only mediate direct cytostatic or cytotoxic effects on cancer cells, but also engage immunological mechanisms such as antibody-dependent cellular cytotoxicity (ADCC), which likely contributes to their efficacy. ${ }^{172}$ Interestingly, EGFR-targeting antibodies were shown to induce ICD and facilitate the elicitation of antitumor $\mathrm{T}$ cells in mouse models, ${ }^{173,174}$ and the clinically relevant antibodies cetuximab and panitumumab induced ICD via a mechanism dependent on the mutational status of the EGFR signaling pathway and involving inhibition of the unfolded protein response, leading to ER stress, CALR translocation to the cell surface, and phagocytosis of the cancer cells by DCs. ${ }^{173}$ Trastuzumab in combination with chemotherapy induced humoral responses to the intracellular domain of HER2 and other tumor-associated antigens, including carcinoembryonic antigen (CEA) and p53, in breast cancer patients, an effect that was associated with improved survival. ${ }^{175}$ Trastuzumab-mediated ADCC was enhanced when conjugated to an anthracycline derivative, an effect that was linked to off-target immunostimulatory effects and synergy with an anti-PD-1 antibody in a murine orthotopic breast cancer model resistant to other HER2-targeted therapies. ${ }^{176}$ Cetuximab has also been attributed offtarget immunostimulatory effects by activating human $\mathrm{CD}^{+} \mathrm{T}$ and NK cells in patients with colorectal cancer, correlating with $\mathrm{T}_{\text {reg }}$ cell inhibition, ${ }^{177}$ as well as systemic effects linked to improved infiltration of liver metastases in colorectal cancer patients. ${ }^{178}$

Epigenetic modifiers are another group of therapeutics with potentially important effects on antitumor immunity. For instance, decitabine, an inhibitor of DNA methyltransferases, has been reported to upregulate interferon signaling, antigen processing and presentation, and cytokine/chemokine-dependent signal transduction, as well as cancer-testis antigen expression in breast, colorectal, and ovarian cancer cells. ${ }^{111}$ These data were confirmed in human samples from patients with colorectal and ovarian cancer responding to lowdose decitabine. ${ }^{179}$ Such a response was attributed to the upregulation of hypermethylated endogenous retroviruses coupled to cytosolic sensing of double-stranded (ds) RNA by TLR3 and mitochondrial antiviral signaling protein. ${ }^{180}$ The antitumor activity of histone deacetylase (HDAC) inhibitor trichostatin A, which is similar to the U.S. Food and Drug Administration (FDA)-approved HDAC inhibitor vorinostat, was shown to depend (at least in part) on the inhibition of intratumoral $\mathrm{CD} 4^{+} \mathrm{T}$ cell apoptosis via downregulation of FAS ligand. ${ }^{181}$

DDR targeting can also mediate immunostimulatory effects and several reports are evaluating the therapeutic efficacy of combining these agents with immunotherapy. ${ }^{87}$ For example, the FDA-approved poly(ADP-ribose) polymerase 1 inhibitors olaparib and rucaparib showed on-target immunostimulatory effects by inducing the accumulation of single-stranded (ss) DNA and micronuclei into the cytosol of NSCLC and triple-negative breast cancer cells. ${ }^{182,183}$ In turn, this activated CGAS and consequent type I IFN secretion (linked to increased PD-L1 expression) and recruitment of $\mathrm{CD}^{+} \mathrm{T}$ cells into the tumor. ${ }^{182,183}$ Moreover, coadministration of ICBs led to superior disease control in tumors with DDR deficiency (including tumors with BRCA1 or BRCA2 mutations). ${ }^{184}$

Cyclin-dependent kinase 4 (CDK4) and CDK6 are frequently upregulated in human malignant cells. ${ }^{185}$ These proteins are involved in cell-cycle regulation and can be specifically targeted with CDK4/6 inhibitors, including palbociclib, ribociclib, and abemaciclib, all of which have recently been approved for the treatment of patients with hormone receptor (HR)-positive breast cancer. ${ }^{186}$ Their therapeutic activity has been largely attributed to their ability to induce cell-cycle arrest in malignant cells, but accumulating reports also highlighted a role for cellcycle inhibitors in promoting on-target and off-target immunostimulatory effects. ${ }^{187}$ In particular, CDK4/6 inhibition can induce on-target effects by enhancing antigen presentation via MHC-I on breast and colorectal cells. ${ }^{188,189}$ Malignant cells treated with CDK4/6 inhibitors can also secrete type III IFN plus multiple inflammatory cytokines as a part of the senescence-associated secretory phenotype (SASP), ${ }^{188,189}$ resulting in the modulation of 
anticancer immune responses. ${ }^{190}$ Moreover, CDK4/6 inhibitors can induce off-target immunostimulatory effects by promoting effector $\mathrm{T}$ cell proliferation upon nuclear factor of activated $\mathrm{T}$ cell 1 activation and downregulating $\mathrm{T}_{\text {reg }}$ cell proliferation. ${ }^{188,189,191}$ Recent findings also demonstrated that the combination of palbociclib with a MEK inhibitor (trametinib) favors a SASP that recruits NK cells with anticancer activity or favors a vascular remodeling that enables CD8 ${ }^{+}$CTL infiltration in preclinical models of KRAS-driven lung or pancreatic adenocarcinomas, respectively. ${ }^{192,193}$ Enhanced T cell activation downstream of genome destabilization has been recently documented for pharmacological CDK7 inhibitors. ${ }^{194}$ Moreover, targeting CDK8 resulted in NK cell-dependent antitumor responses in mice bearing B16F10 melanoma. ${ }^{195}$ Finally, superior tumor eradication has been observed in different mouse tumor models following coadministration of cell-cycle inhibitors with ICBs. ${ }^{188,189,191,193,194,196,197}$

\section{Radiation Therapy}

Radiation has been known for a long time to have dose-dependent pro-inflammatory effects. ${ }^{198}$ In fact, the multifraction radiation regimens traditionally used in clinical practice, which consist of small daily doses given for several weeks (1.5-2.2 Gy/day to a total dose of 50-80 Gy depending on the tumor type), were developed to minimize inflammatory reactions in normal tissue incorporated in the radiation field to account for differences in the daily reproducibility of the patient position. Technological advances have dramatically expanded the range of doses that can be safely delivered to the tumor with each fraction by improving the precision of tumor targeting with stereotactic body radiotherapy (SBRT). 2,199 One or a few high radiation doses (above 8 Gy and up to $30 \mathrm{~Gy}$ ) are used to "ablate" the tumor by increasing the amount of cancer cell death. However, experimental evidence shows that the ability of radiation to activate antitumor immunity is an important determinant of radiation response, ${ }^{200,201}$ and hence that the quality of cancer cell death, namely the induction of ICD, may be more important than its quantity. ${ }^{10,202}$ In some preclinical studies, the local response to radiation given at doses in the SBRT range (single dose of 20-30 Gy) was largely dependent on $\mathrm{T}$ cells and a single large dose was more effective at inducing antitumor T cells than several small doses, ${ }^{11,203}$ but other studies reported activation of antitumor $\mathrm{T}$ cells with a few comparatively lower dose fractions (e.g., 2 Gy X 5, 3 Gy X 5, or 8 Gy X 3), ${ }^{204-206}$ suggesting some degree of model-dependency in this regard..$^{207}$

Overall, the influence of the dose and fractionation regimen on the immunogenic effects of radiation therapy remains incompletely understood. The direct comparison of different radiation doses and fractionation schedules in a few mouse tumor models showed that hypofractionated regimens of 8 Gy X 3 or 6 Gy X 5 (but not single-dose radiation of 20 or $30 \mathrm{~Gy}$ ) were able to induce a potent immune response leading to systemic tumor regression when combined with ICBs. ${ }^{206,208}$ However, when the immunogenicity of radiation is assessed by the immunological control of the tumor in experimental models, several factors (many of which are model-specific) may contribute to outcome, including intrinsic radiosensitivity, immune contexture, and the type of immunotherapy combined with radiation. ${ }^{209}$

Besides contributing to the control of the irradiated lesion, radiation-induced antitumor $\mathrm{T}$ cells can reject metastases outside of the radiation field, a phenomenon known as abscopal effect. ${ }^{207,210-212}$ Tumor responses in non-irradiated metastases have been reported in patients receiving radiation alone but are very rare. ${ }^{213,214}$ However, when radiation was used in patients treated with immunotherapy, either to palliate progressing tumors or in the setting of clinical trials testing combinations of radiation and immune modulators, abscopal responses were detected at increased frequency. ${ }^{215-222}$ The ability of focal tumor radiotherapy in combination with ICBs to induce abscopal responses in patients with NSCLC has been tested prospectively in various studies. In patients with chemorefractory metastatic NSCLC, which is poorly sensitive to ICBs targeting CTLA-4 used alone or combined with chemotherapy, ${ }^{223}$ fractionated radiation (6 Gy X 5 and 9.5 Gy X 5) combined with the anti-CTLA-4 agent ipilimumab induced objective abscopal responses in $18 \%$ of individuals, accompanied by evidence of tumor-specific CD8 ${ }^{+} \mathrm{T}$ cell responses. ${ }^{219} \mathrm{In}$ another study, metastatic NSCLC patients were randomized to receive the anti-PD1 agent pembrolizumab alone or combined with focal radiation (8 Gy X 3), and the latter treatment was associated with superior overall response rate and median overall survival, ${ }^{224}$ supporting the hypothesis that local radiotherapy can play a role in metastatic disease by sensitizing tumors to immunotherapy. ${ }^{8}$ However, results have not been conclusive across different clinical studies, ${ }^{225-228}$ highlighting the need to improve the current understanding of the immunological effects of radiotherapy.

Overall, radiation seems to mediate both on-target and off-target immunomodulatory effects that influence its therapeutic activity and identify novel targets for the development of combinatorial treatment regimens with superior clinical efficacy (Figure 29.2).

\section{Immunostimulatory Effects of Radiation}

\section{Increased Antigenicity}

Multiple studies have shown that radiation enhances the expression of MHC-I molecules as well as some tumor antigens in mouse and human cancer cells maintained in vitro. ${ }^{229,230}$ In one study analyzing the 


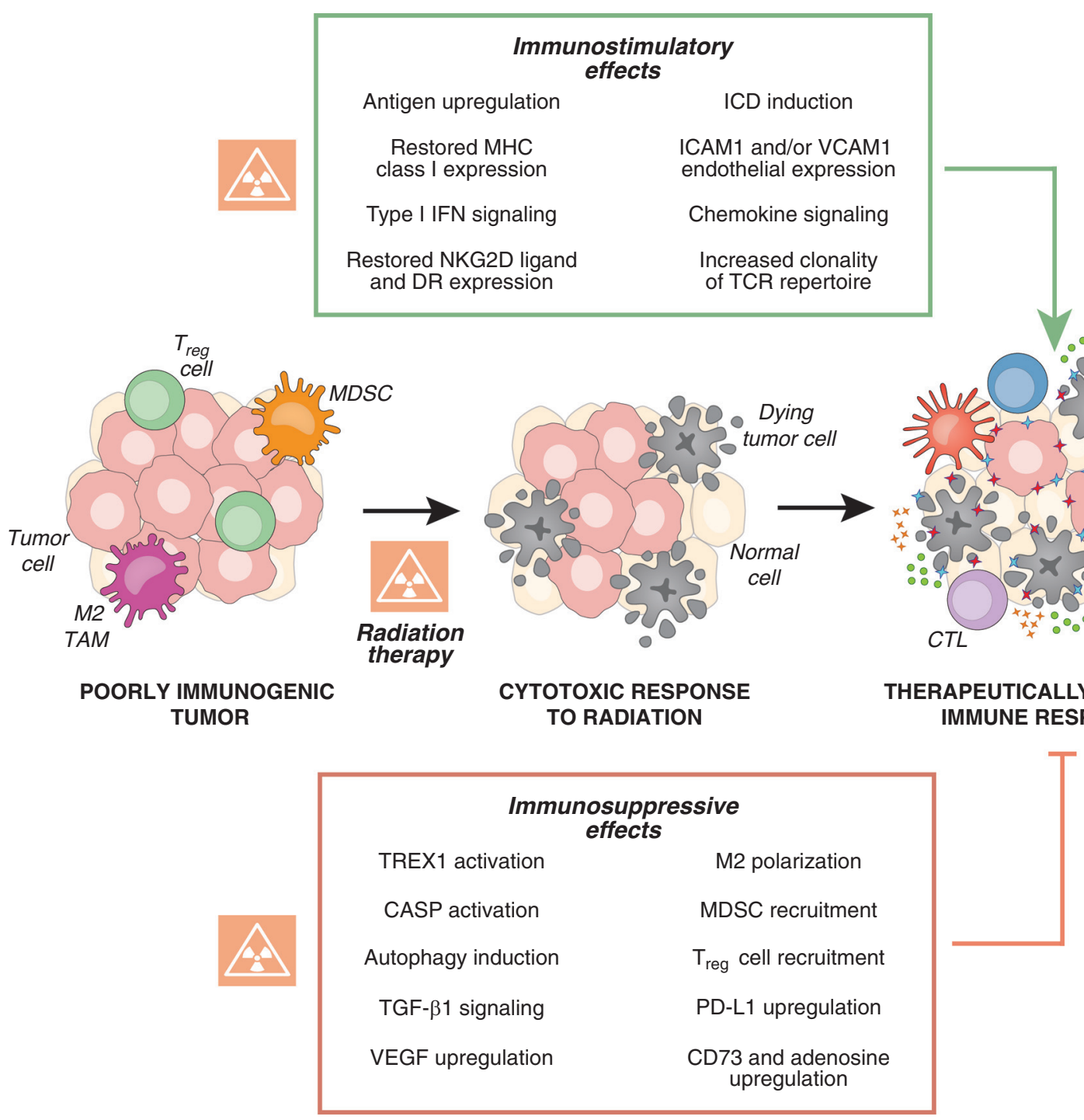

Figure 29.2 Immunostimulation and immunosuppression by radiation therapy. Malignant cells can die in response to radiation therapy as a consequence of irreparable damage to macromolecules and organelles. Importantly, such a cytotoxic response only affects a fraction of the tumor, implying that it is not sufficient to eradicate established neoplasms. Alongside, radiation therapy also mediates immunostimulatory (A) and immunosuppressive (B) effects, originating not only within cancer cells, but also within the stromal, endothelial, and immunological tumor compartments. The balance between such immunostimulatory and immunosuppressive effects determines whether radiation therapy can ultimately stimulate a therapeutically relevant anticancer immune response resulting in tumor eradication.

CD73 (official name: NT5E), 5'-nucleotidase ecto; CTL, CD8+ cytotoxic lymphocyte; DAMP, damage-associated molecular pattern; DC, dendritic cell; DR, death receptor; ICAM1, intercellular adhesion molecule 1; ICD, immunogenic cell death; IFN, interferon; MDSC, myeloid-derived suppressor cell; NK, natural killer; NKG2D (official name KLRK1), killer cell lectin-like receptor K1; PD-L1 (official name, CD274); TAA, tumorassociated antigen; TAM, tumor-associated macrophage; TGF 31 , transforming growth factor $\beta 1 ; T_{\text {reg; }}$ CD4 ${ }^{+} C D 25^{+}$Foxp3 ${ }^{+}$regulatory T; TREX1, three prime repair exonuclease 1; VCAM1, vascular cell adhesion molecule 1; VEGF, vascular endothelial growth factor.

effects of radiation on expression of MHC-I and two tumor antigens, that is, CEA and mucin-1 (MUC-1), exposure to a single radiation dose of 10 or $20 \mathrm{~Gy}$ induced the upregulation of MHC-I and MUC-1 in eight of 23 human colon, lung, and prostate carcinoma cell lines tested, and CEA in 16 of them. ${ }^{28}$ In mouse colorectal carcinoma MC38 cells growing in syngeneic immunocompetent mice, MHC-I upregulation was shown to occur in vivo after a single 10 Gy radiation dose, improving tumor rejection by adoptively transferred tumor-specific CTLs. ${ }^{231}$ Similar findings have been obtained in human NSCLC A549 and H1975 cell lines maintained in vitro, with a plateau effect at $20 \mathrm{~Gy}$ single-dose radiation. ${ }^{232}$

In another study, the mechanisms of radiationinduced MHC-I upregulation were investigated using 
human melanoma cells. ${ }^{231}$ Upregulation was detected at doses of $4 \mathrm{~Gy}$ or above and plateaued between 10 and $25 \mathrm{~Gy}$. In the first few hours postradiation (clean-up phase), increased expression of surface MHC-I was supported by degradation of cellular proteins damaged by reactive oxygen species (ROS) generated by radiation, resulting in a larger pool of peptides available for loading onto MHC-I molecules. In a second phase (repair phase), activation of mechanistic target of rapamycin was required to sustain the increased availability of antigenic peptides, which largely originated from overall enhanced protein synthesis resulting in enhanced generation of defective ribosomal products. Importantly, some peptides displayed on surface MHC-I molecules by irradiated cells were not detected in the immunopeptidome of non-irradiated cells, and originated from proteins synthesized in response to DNA damage. ${ }^{231}$ These data suggest that radiation modulates cancer cell antigenicity by altering the repertoire of epitopes displayed by MHC-I. This may result in increased expression of genes that encode immunogenic mutations, thus exposing neoantigens to the immune system. ${ }^{230}$ Evidence in support of this hypothesis comes from the identification of $\mathrm{CD} 8^{+} \mathrm{T}$ cell clones specific for an immunogenic mutation encoded in karyopherin subunit alpha 2 (KPNA2), a gene upregulated by radiation, in an NSCLC patient with complete response to radiation and ipilimumab. ${ }^{219}$

The upregulation of MHC-I in vivo is not always the result of an on-target effect of radiation. For instance, in the B16 mouse melanoma model, host-produced IFN- $\gamma$ was required for MHC-I upregulation in tumors treated with a single dose of $15 \mathrm{~Gy}{ }^{233}$ This effect has also been observed in an orthotopic HNSCC murine model in which a single radiation dose of 10 Gy promoted IFN- $\gamma$ secretion by $\mathrm{CD}^{+}$and $\mathrm{CD} 8^{+} \mathrm{T}$ cells, ultimately increasing tumor immunogenicity. ${ }^{234}$

Regardless of the mechanisms involved, which include a stable increase in expression of MHC-I heavy chain mRNA in mouse melanoma cells after prolonged exposure to 2 Gy daily radiation doses, ${ }^{235}$ the increased antigenicity of irradiated cancer cells can contribute to their ability to serve as vaccines, especially when doses that are not ablative but may sensitize tumor cells to CTLs are used. In fact, in a mouse intracranial GL261 glioma model, peripheral vaccination with irradiated GM-CSF-transduced GL261 tumor cells had little effect, and whole brain radiotherapy with two doses of $4 \mathrm{~Gy}$ only minimally extended survival. However, when combined, the two treatments cured $40 \%$ to $80 \%$ of the mice. ${ }^{236}$ Interestingly, restored MHC-I expression was seen in invading glioma cells at the edge of the tumor growing in the irradiated brain, suggesting that it may have contributed to complete tumor elimination by vaccine-elicited $\mathrm{T}$ cells.

\section{Increased Adjuvanticity}

Similar to chemotherapy, radiation has been shown to increase the capacity of malignant cells to emit danger signals. Cell stress-associated danger signals expressed on cells that survive irradiation include several NKG2D ligands, death receptors, adhesion molecules, and co-stimulatory molecules. ${ }^{237}$ Ionizing radiation induced the expression of NKG2D ligands ULBP1 and RAET1E in mouse ovarian cancer cells, ${ }^{238}$ and MICB, ULBP1, and ULBP2 in several human cancer cells, increasing their sensitivity to NK cell-mediated lysis. ${ }^{239}$ Radiation also promoted the immunogenicity of different glioma cell lines, including glioma stem-like cells, in vitro and in vivo, by upregulating the expression of several NKG2D ligands at the mRNA and protein levels and enhancing DDR. ${ }^{240}$ Consistently, decreased NKG2D ligand expression coupled to increased PD-L1 expression has been found in radioresistant NSCLC cell lines, protecting these cells from the cytotoxic action of NK cells. ${ }^{241}$ Sensitivity of cancer cells to NK cell-mediated lysis was also increased by radiation-induced release of diablo IAP-binding mitochondrial protein (DIABLO) from mitochondria, which increases granzyme-induced cytotoxicity by blocking X-linked inhibitor of apoptosis (XIAP). ${ }^{242}$ Moreover, inhibition of XIAP has been related to enhanced radiation-induced antigen presentation, induction of ICD, and recruitment of T cells in mouse models of HNSCC. ${ }^{243}$ In the $4 \mathrm{~T} 1$ mouse breast cancer model, in vivo irradiation increased the expression of intercellular adhesion molecule 1 (ICAM1) and induced the exposure of RAET1E on the surface of cancer cells. The interaction of RAET1E with NKG2D expressed on $\mathrm{CD} 8^{+} \mathrm{T}$ cells was critical for formation of stable immune synapses between these immune effectors and cancer cells in mice treated with an anti-CTLA-4 antibody. ${ }^{244}$ Expression of death receptor FAS/CD95 was induced in MC38 colorectal mouse tumor cells after in vivo irradiation with a single $8 \mathrm{~Gy}$ radiation dose and increased tumor rejection by CD8 ${ }^{+} \mathrm{T}$ cells. ${ }^{245}$ Similar findings have been obtained in models of pancreatic adenocarcinoma treated with CAR T cells. ${ }^{246}$ In this setting, low-dose (1-2 Gy) irradiation caused the upregulation of TNF receptor superfamily member 10b (TNFRSF10B, best known as DR5) on the surface of malignant cells, ultimately resulting in some degree of antigen-independent cytotoxicity by CAR T cells. ${ }^{246}$ Finally, irradiation of human colorectal cancer cells in vitro resulted in the upregulation of OX40L and 41BBL through epigenetic modulation, resulting in improved CTL survival, activation, and effector functions. ${ }^{247}$

Radiation has also been shown to induce the expression and release of many pro-inflammatory cytokines and chemokines by cancer cells. In vitro, a single radiation dose of $20 \mathrm{~Gy}$ induced IL-1 $\beta$ release by leukemia cells. ${ }^{248}$ Human sarcoma cells produce TNF in response 
to a single radiation dose of $5 \mathrm{~Gy} .{ }^{249} \mathrm{CXCL} 16$ was induced in vitro in human breast cancer cells and murine mammary, prostate, and colon carcinoma cells by a single dose of 12 Gy. ${ }^{250,251}$ Moreover, radiation-induced CXCL16 was required for optimal infiltration of $4 \mathrm{~T} 1$ mouse breast tumors by activated $\mathrm{CD} 8^{+} \mathrm{T}$ cells in mice treated with anti-CTLA-4 antibody. ${ }^{250}$ Other than CXCL16, CXCL10, CCL2, and CCL5 also increased in B16 melanoma tumors after 30 Gy of radiation given in two fractions, resulting in increased $\mathrm{CD} 8^{+} \mathrm{T}$ cells and decreased $\mathrm{CD} 11 \mathrm{~b}^{+} \mathrm{Gr} 1^{+}$ MDSCs tumor infiltration. ${ }^{252}$

Importantly, radiation-induced cancer cell death is associated with the generation of at least some of the DAMPs that characterize ICD induced by chemotherapy: CALR translocation to the cell surface, ATP secretion, and HMGB1 release, all of which have been shown to manifest in a radiation dose-dependent manner. ${ }^{53,58,218,253,254}$ In addition, DNA released by cancer cells succumbing to radiation in vivo can be taken up by tumor-infiltrating DCs and provide a critical signal by activating IFN-I production via CGAS and stimulator of interferon response cGAMP interactor 1 (STING1), ${ }^{255}$ an effect that may depend, at least in part, on tumor-derived exosomes. ${ }^{256}$ At least in some settings, STING1 activation and consequent IFN-I production also occurs in irradiated cancer cells downstream of the accumulation of double-stranded (ds) DNA in the cytosol. ${ }^{257}$ The ability of cancer cells to produce IFN-I is dependent on multiple factors, including expression levels of CGAS and STING1, the status of the cofactors and posttranslational modifications that modulate their functions, and the radiation dose and fractionation schedule. ${ }^{258}$ One of the regulatory mechanisms limiting the secretion of IFN-I by irradiated cells is mediated by three prime repair exonuclease 1 (TREX1), which degrades cytosolic dsDNA and is upregulated at least in some cancer cells by radiation doses above 12 Gy. Conversely, production of IFN-I following CGAS-STING1 activation appears to be optimal when radiation is given in hypofractionated doses (8 Gy X 3). Upregulation of IFN-inducible chemokines (i.e., CXCL9, CXCL10, CXCL11, and CXCL16) and consequent recruitment of conventional type 1 DCs $(\mathrm{cDC} 1 \mathrm{~s})$ and effector $\mathrm{CD}^{+} \mathrm{T}$ cells to the irradiated tumor were also seen in mouse tumors following fractionated radiation doses that optimally induce IFN-I. ${ }^{206}$ Thus, in vivo radiation amplifies a pathway responsible for priming of spontaneous antitumor immunity to immunogenic tumors. ${ }^{10,259}$

\section{Off-Target Immunostimulatory Effects}

Some of the stromal cells present within the irradiated tumor have been shown to respond to radiation with phenotypic changes that improve tumor infiltration of effector T cells. Most of the studies have analyzed changes in tumor endothelium, showing upregulation of vascular cell adhesion molecule (VCAM1), which was associated with increased tumor-specific $\mathrm{CD} 8^{+} \mathrm{T}$ cell infiltration into irradiated mouse B16 melanomas. ${ }^{204}$ Ionizing radiation also induced ICAM1 and VCAM1 expression on lymphatic endothelial cells in MC38 and B16 tumors grown in mice, an effect that was partially mediated by TGF- $\beta 1 .{ }^{260}$ In another study based on a mouse model of spontaneous pancreatic islet carcinogenesis, radiation used at a low dose of 0.5 to 2 Gy normalized the aberrant vasculature by re-programming macrophages into a M1 phenotype, resulting in endothelial activation and recruitment of adoptively transferred T cells. ${ }^{261}$ Interestingly, a recent study using longitudinal in vivo imaging of mouse tumors demonstrated that a significant fraction of tumor-infiltrating T cells survives therapeutic doses of radiation and exhibits increased motility and cytokine secretion as compared to $\mathrm{T}$ cells from unirradiated tumors. ${ }^{13}$ These data indicate that, unlike circulating naïve $T$ cells, tumor-resident $T$ cells respond to radiation with increased effector functions rather than apoptosis.

Overall, the previously described multiple immune activating signals elicited by radiation explain the ability of radiation to convert the tumor into an "in situ vaccine" and induce antitumor immune responses that have been shown to contribute to control of the irradiated tumor in experimental studies. ${ }^{200,262}$ However, the immune activation is usually insufficient to overcome negative regulatory networks that can also be upregulated by radiation, precluding the development of systemic responses capable of mediating abscopal effects. This could also be because radiation dose and fractionation schedules for inducing the optimal antitumor immune response and for reducing toxicity are not well defined and may vary between tumors with different genomic alterations and immune contextures. ${ }^{199}$ Clearly, evidence for development of systemically detectable antitumor T cells in radiotherapy-treated patients is tenuous, ${ }^{263}$ and abscopal responses are rarely seen in patients, as well as in tumor-bearing mice treated with radiation alone, ${ }^{209}$ suggesting that the immunostimulatory effects of radiation are often insufficient to generate robust antitumor immune responses that overcome existing and radiation-driven immunosuppressive barriers.

\section{Immunosuppressive Effects of Radiation}

Radiation causes DNA damage that activates several stress responses including a cytoprotective DDR. ${ }^{264}$ As previously described, the removal of cytosolic DNA fragments by TREX1 can limit the secretion of type I IFN by cancer cells. ${ }^{206}$ In this context, ATR serine/threonine kinase (ATR), a regulator of the DDR, has been shown to decrease IFN-I secretion by cancer cells, PD-L1 upregulation on tumor cells, and $\mathrm{CD}^{+} \mathrm{T}$ cell exhaustion. Enhancing DNA damage and accumulation of micronuclei by ATR pharmacological inhibition in combination 
with radiation significantly increased radiation-mediated IFN-I responses and MHC-I antigen presentation in lung cancer cells, and potentiated CD8 ${ }^{+} \mathrm{T}$ cell activity in mouse models of KRAS-mutant cancer. ${ }^{265-267}$ Another DNA damage regulator, ATM serine/threonine kinase (ATM), has been shown to activate NF- $\mathrm{BB}$ signaling pathway in radioresistant cancer cells, ${ }^{268}$ resulting in the secretion of pro-inflammatory cytokines but limited STING-mediated type I IFN production. ${ }^{26,270}$ As a consequence of DDR or ROS generation by damaged mitochondria, cancer cells can also activate autophagy as a mechanism of defense in response to radiation, ${ }^{202,264}$ and radiation-induced autophagy can act as an immunosuppressive mechanism by removing the cytosolic sources of dsDNA including micronuclei ${ }^{271,272}$ and limiting CGAS-STING pathway activation. ${ }^{273}$ Moreover, CGAS-STING-dependent type I IFN production can be prevented by caspase 9 (CASP9) and CASP3, both of which are activated in cells succumbing to radiation-driven ICD. ${ }^{5,264,274,275}$ In particular, both Casp9-- and Casp3 ${ }^{--}$cells growing in immunocompetent mice are more sensitive to radiation and generated a superior abscopal response in combination with ICBs. ${ }^{212,275,276}$ In addition, CASP3 mediated prostaglandin E2 release by irradiated cancer cells, which is known to favor the establishment of an immunosuppressive tumor microenvironment. ${ }^{277}$

Radiation has been shown to upregulate expression of a number of growth factors and chemokines that promote the influx of myeloid cells into the tumor and foster their differentiation into immunosuppressive and tumor-promoting MDSCs and/or M2-polarized TAMs. ${ }^{32}$ In the $\mathrm{MycCaP}$ mouse model of prostate carcinoma, radiation increased CSF1 release by cancer cells, leading to a systemic increase in circulating MDSCs, which were recruited to the tumor. Response of the irradiated tumor was improved by administration of an inhibitor of CSF1R. Increased levels of CSF1 were also found in the serum of prostate cancer patients after radiotherapy. ${ }^{278} \mathrm{In}$ a model of pancreatic ductal adenocarcinoma, radiation upregulated the chemokine CCL2, which increased the recruitment of $\mathrm{Ly}_{6} \mathrm{C}^{+} \mathrm{CCR} 2^{+}$ monocytes and their differentiation into pro-tumorigenic and pro-angiogenic TAMs. ${ }^{279}$ Induction of TAMs in irradiated pancreatic tumors in mice also correlated to fewer $\mathrm{CD} 8^{+} \mathrm{T}$ cells and increased numbers of effector $\mathrm{CD} 4^{+} \mathrm{T}$ cells and $\mathrm{T}_{\text {reg }}$ cells. ${ }^{280}$ Genetic or antibody-mediated CCL2 blockade, neutralization of macrophage colony-stimulating factor, and treatment with anti-CCR2 antibody alleviated immunosuppression, improving tumor response to radiation. ${ }^{279-281}$ Interestingly, CCL2 was not induced by radiation in $\mathrm{MycCaP}$ prostate carcinomas, indicating that different tumors rely on different pathways to recruit myeloid cells in response to radiation. ${ }^{278}$ Another radiation-induced pathway that promotes immunosuppression involves the upregulation of hypoxia-inducible factor 1 and consequently vascular endothelial growth factor A (VEGFA), ${ }^{282}$ which promotes tumor infiltration by MDSCs and $\mathrm{T}_{\text {reg }}$ cells. ${ }^{283} \mathrm{In}$ vivo treatment with an aptamer platform that targets radiation-induced VEGFA and 4-1BB (an immune-stimulatory receptor expressed on activated $\mathrm{CD} 8^{+} \mathrm{T}$ cells) potentiated both local tumor control and abscopal responses and reduced tumor-infiltrating $\mathrm{T}_{\text {reg }}$ cells. ${ }^{284} \mathrm{~T}_{\text {reg }}$ cells have also been shown to be more radioresistant than conventional $\mathrm{T}$ cells, resulting in a relative increase postradiation, ${ }^{28,286}$ although tumor-infiltrating effector T cells also appear to be more radioresistant than their circulating counterparts. ${ }^{13}$ Moreover, radiation promotes the expansion and enhances the suppressive function of suppressive tumor-infiltrating $\mathrm{T}_{\text {reg }}$ cells, characterized by higher expression of CTLA-4, CD137, and Helios, in several murine tumor models (B16/ F10, RENCA, and MC38). ${ }^{287} \mathrm{~T}_{\text {reg }}$ cell depletion improved tumor control achieved by radiation in different cancer models. ${ }^{28,289}$ Finally, STAT3 inhibition decreased $\mathrm{T}_{\text {reg }}$ cells, MDSCs, and M2 macrophages in response to radiotherapy, but enhanced effector T cells and M1 macrophages, improving tumor growth delay in preclinical models of HNSCC. ${ }^{289}$

Many tumors are rich in TGF- $\beta 1$ but the vast majority is bound to latency-associated peptide (LAP) and inactive. Radiation-induced ROS causes TGF- $\beta 1$ dissociation from LAP and its activation, ${ }^{290}$ an event that plays a major role in inhibiting DC activation and priming of tumor-specific $\mathrm{T}$ cells. When TGF- $\beta 1$ was neutralized by an antibody, radiation elicited $\mathrm{T}$ cell responses to multiple tumor antigens and abscopal effects in mouse models of metastatic breast cancer. ${ }^{291}$ Abscopal responses were not documented in a prospective randomized clinical study testing systemic TGF- $\beta$ blockade (fresolimumab) in two doses in the context of local hypofractionated radiation in a cohort of 23 patients with metastatic breast cancer. However, patients receiving the higher dose of fresolimumab showed changes consistent with decreased immunosuppression and improved $\mathrm{CD} 8^{+} \mathrm{T}$ cell memory and experienced longer median overall survival compared to the patients treated with the lower fresolimumab dose. ${ }^{292}$

In vivo upregulation of PD-L1 by radiation has been reported in cancer cells and infiltrating myeloid cells in murine tumors. ${ }^{205,234,241,291,293}$ In most cases, PD-L1 upregulation was driven by IFN- $\gamma$ produced by radiation-induced antitumor $\mathrm{T}$ cells and acted as a barrier to tumor rejection. ${ }^{205,234}$ However, CASP9 activation in irradiated cells has also been mechanistically linked to PD-L1 upregulation. ${ }^{275}$ Other than PD-L1, the adenosine-generating enzyme CD73 and adenosine are upregulated in irradiated mouse and human breast cancer cells. ${ }^{294}$ The CD73-adenosine axis on tumor cells is well known to impair antitumor $\mathrm{T}$ cell responses $^{295}$ and CD73 blockade with radiotherapy was able to restore DC infiltration in settings in which induction of IFN-I by radiotherapy alone was suboptimal. ${ }^{294}$

Other factors that may exert an immunosuppressive effect in the context of some tumors include the complement system, which was activated in a mouse model 
of lymphoma and mediated fast clearance of apoptotic cells reducing the generation of inflammatory signals, ${ }^{296}$ as well as galectin-1 (as demonstrated in a mouse lung carcinoma model) ${ }^{297}$ In summary, radiation can elicit a variety of immunosuppressive mechanisms that, at least potentially, limit its therapeutic efficacy and thus offer valuable targets for the development of combinatorial therapeutic regimens (Figure 29.2).

\section{POTENTIAL ROLE OF THE GENETIC BACKGROUND OF THE HOST}

Accumulating clinical data indicate that the genetic background of cancer patients can influence disease outcome as it influences the ability of malignant cells to elicit an immune response following chemotherapy, targeted therapy, or radiation therapy. ${ }^{298}$ Loss-of-function single nucleotide polymorphisms (SNPs) affecting TLR3 (encoding the receptor for cancer cell-derived ssRNA), TLR4 (encoding the receptor for extracellular HMGB1), $P 2 R X 7$ (encoding the immunostimulatory receptor for extracellular ATP), and FRP1 (encoding the receptor for extracellular ANXA1) have all been associated with reduced disease-free or overall survival in cohorts of breast carcinoma patients treated with neoadjuvant anthracycline-based chemotherapy ${ }^{55,57,58,299}$ Along similar lines, SNPs in FPR1 and TLR4 have been linked to poor disease outcome in two independent cohorts of colorectal carcinoma patients. ${ }^{299,300}$ Moreover, a genetic variant of advanced glycosylation end-product specific receptor (AGER; another HMGB1 receptor best known as RAGE) has been associated with poor response to chemotherapy among NSCLC patients. ${ }^{301}$ Finally, SNPs in IDO1 and CD24 (encoding an adhesion molecule involved in the interaction between DCs and T cells) have been linked to decreased 5-year survival rate in patients with resected colorectal liver metastases. ${ }^{302}$ Altogether, these observations exemplify the potential effect of the genetic background of the host on the elicitation of anticancer immune responses by different treatment modalities.

\section{CONCLUSION}

Growing evidence indicates that the therapeutic effect of cytotoxic treatments is mediated by the interaction of stressed and dying cancer cells with the host immune system. Success likely depends on the balance between activating and suppressive signals generated by treatment, which is determined by the interaction of at least three factors: (1) the effects of therapy on cancer cells and immune cells; (2) the intrinsic immunogenicity of the cancer cells; and (3) germline polymorphisms in key immune players. Moreover, accumulating evidence supports a key role for the gut microbiota and a number of environmental and metabolic factors in the immunological fitness of the host and hence its capacity to mount robust anticancer responses to treatment. ${ }^{303-306}$ Finally, some neoplasms (the so-called "cold tumors") are able to prevent infiltration by immune cells compromising the immunostimulatory effects of chemotherapy, radiotherapy, and targeted therapy, and the efficacy of immunotherapy. ${ }^{5,307}$ To which degree cancer cell death and the disruption of the tumor microenvironment imposed by chemotherapy and/or radiotherapy can turn cold tumors into hot ones remains to be determined.

As previously mentioned, the immunogenicity of a tumor is determined by antigenicity and adjuvanticity. Some tumors are more antigenic due to the presence of large numbers of mutations, some of which will encode neoantigens. ${ }^{16,17}$ Others have mutations in MHC-I genes resulting in irreversible loss of expression. ${ }^{308-312}$ Oncogenic pathways activated in different tumors are emerging as important regulators of tumor adjuvanticity, ${ }^{313,314}$ and polymorphisms in genes encoding DAMP receptors such as TLR4 and P2RX7 affect immune cell activation. ${ }^{7,55,58}$ Each of these factors provides opportunities to improve treatment outcome by devising personalized combinations of agents and ultimately induces a robust and persistent antitumor $\mathrm{T}$ cell memory response that not only contributes to cancer control but provides life-long protection from recurrence in the patient. ${ }^{315}$ Multiple clinical trials testing combinations of immune checkpoint inhibitors and other immunotherapies with chemotherapy, ${ }^{86}$ targeted anticancer agents, ${ }^{316-319}$ or radiotherapy ${ }^{320,321}$ are ongoing. Research to improve our understanding of the immune effects of currently used drugs, agents that are still under development, and the various radiation doses and fractionation regimens employed is urgently needed to accelerate progress and achieve therapeutic success in ever more, and eventually all, cancer patients.

\section{KEY REFERENCES}

Only key references appear in the print edition. The full reference list appears in the digital product on Springer Publishing Connect: connect.springerpub.com/content/book/978-0-8261-3743-2/ part/part02/chapter/ch29

5. Galluzzi L, Chan TA, Kroemer G, et al. The hallmarks of successful anticancer immunotherapy. Sci Transl Med. 2018;10(459):eaat7807. doi:10.1126/scitranslmed.aat7807

14. Rodriguez-Ruiz ME, Vanpouille-Box C, Melero I, et al. Immunological mechanisms responsible for radiation-induced abscopal effect. Trends Immunol. 2018;39(8):644-655. doi:10.1016/j.it.2018.06.001

26. Galluzzi L, Vitale I, Warren S, et al. Consensus guidelines for the definition, detection and interpretation of immunogenic cell death. J Immunother Cancer. 2020;8(1):e000337. doi:10.1136/jitc-2019-000337

219. Formenti SC, Rudqvist NP, Golden E, et al. Radiotherapy induces responses of lung cancer to CTLA-4 blockade. Nat Med. 2018;24(12):1845-1851. doi:10.1038/s41591-018-0232-2

258. McLaughlin M, Patin EC, Pedersen M, et al. Inflammatory microenvironment remodelling by tumour cells after radiotherapy. Nat Rev Cancer. 2020;20(4):203-217. doi:10.1038/ s41568-020-0246-1 